\title{
The Potential of Clove Essential Oil Microemulsion as an Alternative Biocide Against Pseudomonas aeruginosa Biofilm
}

\author{
Isty Adhitya Purwasena (D), Dea Indriani Astuti* (D), Intan Taufik (D) \\ and Fadilla Zahra Putri $\mathbb{D}$
}

Department of Microbiology, School of Life Science and Technology, Institut Teknologi Bandung, Jl. Ganeca No. 10, Lebak Siliwangi - 40132, Bandung, Indonesia.

\begin{abstract}
Biocorrosion phenomenon is known to be detrimental in oil and gas industry. Synthetic biocides have been widely used to eliminate microorganisms which promote biocorrosion. However, these biocides are known to be toxic and harmful to the environment. One of the natural agents that potentially serves as an alternative for biocide is clove essential oil which has antimicrobial constituents and also have been shown to inhibit quorum sensing (QS) process of Pseudomonas aeruginosa. In this study, clove essential oil extracted from clove bud and stem were formulated into microemulsion and evaluated for its antibiofilm and anti-QS activity. To observe its antibiofilm activity, minimum inhibitory concentration (MIC), minimum biofilm inhibitory concentration (MBIC), and minimum biofilm eradicating concentration (MBEC) of clove bud and stem essential oils were determined by microdilution method in microtiter 96 well-plate. Anti-QS activity was observed by analyzing clove bud and stem essential oil effect against $P$. aeruginosa's extracellular protease activity and swarming motility. The MIC value of clove bud and stem essential oils microemulsions were $0,33 \%$ and $0,5 \%$,

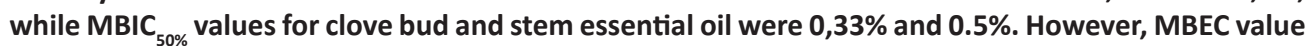
for both clove essential oil could not be determined. In addition, $0.66 \%$ clove bud essential oil and $1 \%$ clove stem essential oil successfully inhibited extracellular protease activity and swarming motility of P. aeruginosa by $32.06 \%$ and $\mathbf{4 5 . 5 6 \%}$ for clove bud, and $31.43 \%$ and $45 \%$ for clove stem, respectively.
\end{abstract}

Keywords: Eugenol, biocorrosion, clove essential oil, MBEC, MBIC, MIC

*Correspondence: dea@sith.itb.ac.id; +62222511575

(Received: December 16, 2019; accepted: March 06, 2020)

Citation: Isty Adhitya Purwasena, Dea Indriani Astuti, Intan Taufik and Fadilla Zahra Putri, The Potential of Clove Essential Oi Microemulsion as an Alternative Biocide against Pseudomonas aeruginosa Biofilm, J. Pure Appl. Microbiol., 2020; 14(1):261-269. https://doi.org/10.22207/JPAM.14.1.27

(c) The Author(s) 2020. Open Access. This article is distributed under the terms of the Creative Commons Attribution 4.0 International License which permits unrestricted use, sharing, distribution, and reproduction in any medium, provided you give appropriate credit to the original author(s) and the source, provide a link to the Creative Commons license, and indicate if changes were made. 


\section{INTRODUCTION}

Corrosion phenomenon have caused detrimental impact in various industrial sector including oil and gas industry. Corrosion causes material and metal degradation which cost structural integrity loss and thickness reduction on equipments. Maintenance process that required to fix the equipments can reduce productivity and increase operational costs on the industry. One of many underlying causes of corrosion is the growth of microorganisms in biofilm that formed on the material surfaces. This phenomenon is known as microbiologically influenced corrosion. Microorganisms involved in this process utilize hydrocarbon or inorganic compounds for metabolism and produce acidic by-products which enhanced corrosion ${ }^{1,2}$. One of biofilm forming bacteria that is widely known to invade oil and gas infrastructure, which then trigger corrosion is Pseudomonas aeruginosa. It also revealed that $P$. aeruginosa is one of major biofilm formation pioneers ${ }^{3}$.

To date, synthetic biocide has been employed to eliminate these microorganisms ${ }^{4}$. However, this treatment is increasingly inexpedient due to its toxicity and harmful effect to the environment. Thus, it requires a safer, non-toxic antimicrobial compound as an alternative to reduce the usage of synthetic biocide.

Essential oils are natural volatile compound that commonly extracted from medicinal plants that have been extensively studied due to their activities as antimicrobial agent ${ }^{5}$. Clove essential oil has been widely recognized as the most prominent aromatic oil based on its pleasant aroma and superior antimicrobial activities ${ }^{6-8}$, which is attributed to eugenol as its major bioactive component (48.8\%$96.00 \%)^{9,10}$. In addition to its antimicrobial agents, clove essential oil has been proven to inhibit the biofilm formation by Pseudomonas aeruginosa as well ${ }^{11,12}$. However, its hydrophobicity and volatility will become a bottle-neck issue when applying essential oil as an antibiofilm. These characteristics might cause essential oil residues unexpectedly converted to gas phase before exposing the targeted biofilm ${ }^{13}$. Microemulsion is expected to be an effective delivery system to increase the exposure between essential oil and biofilm, due to its economically friendly method ${ }^{14}$. Clove essential oil microemulsion has been used to prevent Penicillium digitatum growth in food $^{15}$ and its effect on Fatty Liver and Dyslipidemia has been studied ${ }^{14}$. However, its activity on antibiofilm produced by Pseudomonas aeruginosa has not extensively studied yet.

The main constituent of clove essential oil, which is eugenol has been reported to exhibit antimicrobial and anti-quorum sensing (QS) ability ${ }^{12,16}$. This research investigated the activity of clove essential oil in the form of microemulsion against $P$ seudomonas aeruginosa's biofilm formation and QS process. In addition, the antibiofilm activity of clove essential oil extracted from bud and stem was also compared in this study.

\section{MATERIALS AND METHOD \\ Clove essential oil preparation}

Clove bud and clove stem essential oil were obtained from CV. Karunisa Atsiri Harapan, Surabaya, East Java. Essential oil microemulsion was formulated by mixing clove essential oil, tween 20 and deionized water in 1:6:13 (v/v/v) ratio ${ }^{17}$. Microemulsion stability was tested physically using centrifugation and thermodynamically using freeze thaw and heating cooling cycle. Microemulsion's droplet size was measured with Particle Size Analyzer ${ }^{18}$.

\section{Bacterial isolate preparation}

Pseudomonas aeruginosa isolate was obtained from Microbiology Laboratory in School of Life Science and Technology, Bandung Institute of Technology.

\section{Biofilm formation}

P. aeruginosa's biofilm was grown by incubating $P$. aeruginosa culture at $37^{\circ} \mathrm{C}$ for 12 hours in 96 wells microtiter plate. Biofilm was stained with crystal violet, while the turbidity was measured to indicate the biofilm growth. The measurement was performed using Bio-Rad ELISA Reader at $595 \mathrm{~nm}$ for 12 hours interval ${ }^{19}$.

\section{Minimum Inhbitory Concentration (MIC) assay}

A 20 $\mu$ l aliquot of pre-activated culture was added to $180 \mu$ l of clove essential oil microemulsion in 96-wells microtiter plate. P. aeruginosa culture was mixed with $30 \%$ tween-20 in sterile deionized water and used as negative control. The culture 
growth was measured with Bio-Rad ELISA Reader at $595 \mathrm{~nm}$. Measurement was conducted two times, at the beginning and the end of incubation. Initial measurement was performed before and after 24 hours of incubation at $37^{\circ} \mathrm{C}$. MIC value was expressed as the lowest concentration of clove essential oil needed to inhibit $P$. aeruginosa's planktonic cell growth.

\section{Minimum Biofilm Eradicating Concentration} Assay

A $200 \mu \mathrm{l}$ aliquot of pre-activated culture was incubated at $37^{\circ} \mathrm{C}$ for 48 hours in 96 wells microtiter plate. After incubation, microtiter plate was gently rinsed with Phosphate Buffer Saline (PBS) twice, then added with $200 \mu \mathrm{l}$ of clove essential oil microemulsion and incubated at $37^{\circ} \mathrm{C}$ for 24 hours. After the incubation period, microtiter plate was rinsed with PBS, and air dried for 30 minutes. Then, biofilm was stained with $0.1 \%$ crystal violet. After 30 minutes of incubation, excess crystal violet was removed. Microtiter plate was rinsed with PBS and $20 \mu \mathrm{l}$ of $10 \%$ glacial acetic acid was added to each well. The absorbance of crystal violet stained biofilm was measured using Bio-Rad ELISA Reader at $595 \mathrm{~nm}$. MBEC value was determined when $\geq 50 \%$ biofilm was eradicated $^{19-21}$. MBEC assay was conducted to $P$. aeruginosa biofilm that had been pre-incubated for $36,48,69,72$, and 84 hours.

\section{Minimum Biofilm Inhibitory Concentration}

An aliquot of $100 \mu \mathrm{l}$ culture and $100 \mu \mathrm{l}$ of clove essential oil microemulsion were added to 96-wells microtiter plate and incubated at $37^{\circ} \mathrm{C}$ for
24 hours. After incubation, microtiter plate was rinsed with PBS, stained with crystal violet and suspended in $10 \%$ glacial acetic acid as described in MBEC assay method. MBIC was determined when $\geq 50 \%$ biofilm formation was inhibited $\geq 50 \%^{19-21}$.

\section{Measurement of total extracellular protease} activity

Extracellular protease activity assay was performed using agar diffusion method. $P$. aeruginosa's culture was incubated with clove essential oil microemulsion. After incubation, the culture was centrifuged at $10,000 \mathrm{rpm}$ for 15 minutes to obtain supernatant. Then, filter paper disc was soaked in supernatant, inoculated with skim milk agar and incubated at $37^{\circ} \mathrm{C}$ for 24 hours. Protease activity was observed by measuring the inhibition zone formed on around discs ${ }^{22}$.

\section{Swarming motility activity assay}

This assay was conducted on semisolid medium made from nutrient broth and $0.5 \%$ agar. Agar medium was inoculated with $5 \mu l$ of bacterial culture that was pipetted on to the middle-top of agar surface. Agar medium was incubated at $37^{\circ} \mathrm{C}$ for 24 hours and culture's motility was observed from growth pattern of the bacterial colony. Swarming motility activity was observed by measuring the diameter of bacterial swarming growth ${ }^{23}$.

\section{RESULTS}

Physical stability of microemulsion was tested using centrifugation whereas thermodynamic stability was tested using freeze

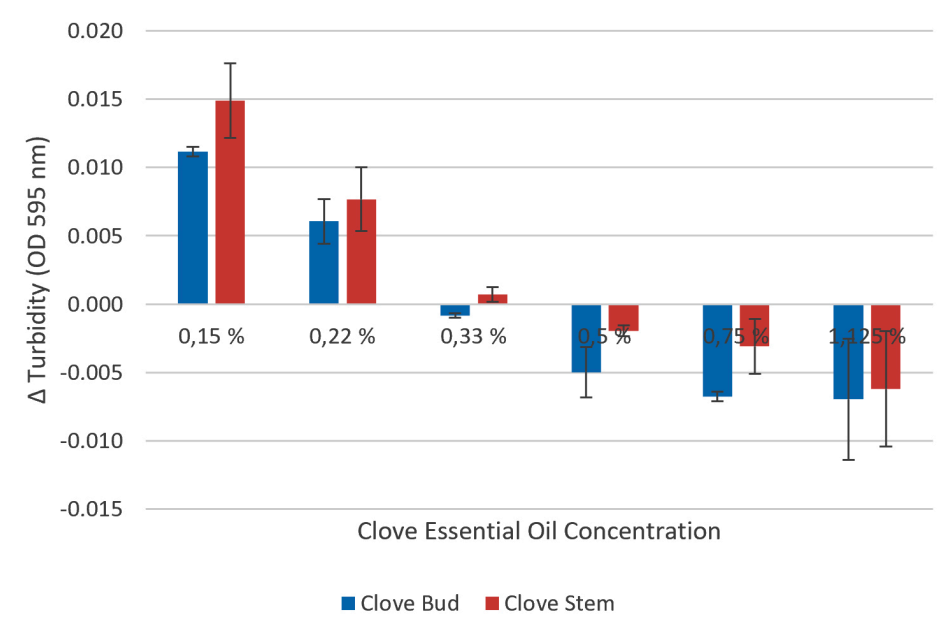

Fig. 1. MIC assay of clove essential oil microemulsion. Clove bud essential oil's MIC was $0.33 \%$, while clove stem essential oil's MIC was $0.5 \%$ 
thaw and heat-cooling cycle. Phase separation and turbidity change which have a P-value higher than 0.05 indicating physical and thermodynamic instability. However, no phase separation and significant turbidity change were observed. This result indicated that the formulated microemulsion was stable both physically and thermodynamically. In addition, droplet size measurement showed that the average diameter of the droplets was less than $100 \mathrm{~nm}$, which confirmed the formulated solution was indeed a micromemulsion ${ }^{14}$.

MIC values for microemulsion of clove essential oil from clove bud and stem are shown in Fig. 1. MIC of clove bud essential oil microemulsion was $0.33 \%$ whereas clove stem essential oil microemulsion was $0.5 \%$.
MBEC values of clove bud and clove stem essential oil are shown in Fig. 2 and 3. The results showed that in the treatment with the highest concentration of essential oil, biofilm elimination occurred less than $60 \%$. Treatment with $0.66 \%$ of clove bud essential oil eliminated $58.82 \%$ biofilm, while $1 \%$ clove stem essential oil treatment eliminated $54.8 \%$ biofilm. Thus, MBEC value for clove bud and clove stem essential oil could not be determined.

Fig. 4 shows the elimination percentage of clove bud and clove stem essential oil microemulsion compared with biofilm growth curve. The highest biofilm elimination occurred to the biofilm that previously pre-incubated for 84 hours and had a low turbidity. Meanwhile, the

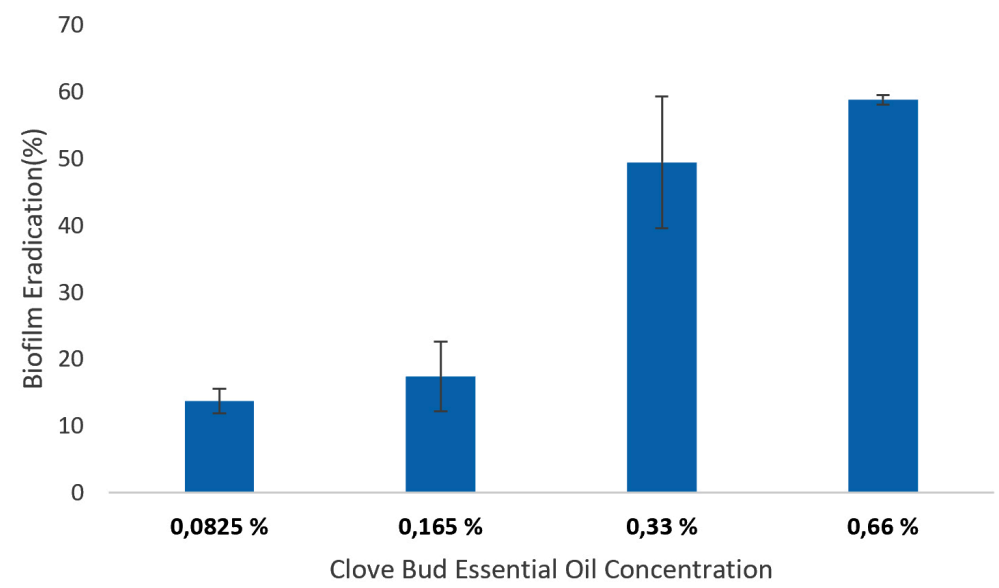

Fig. 2. MBEC assay of clove bud essential oil microemulsion. The highest concentration evaluated did not result in $100 \%$ biofilm elimination

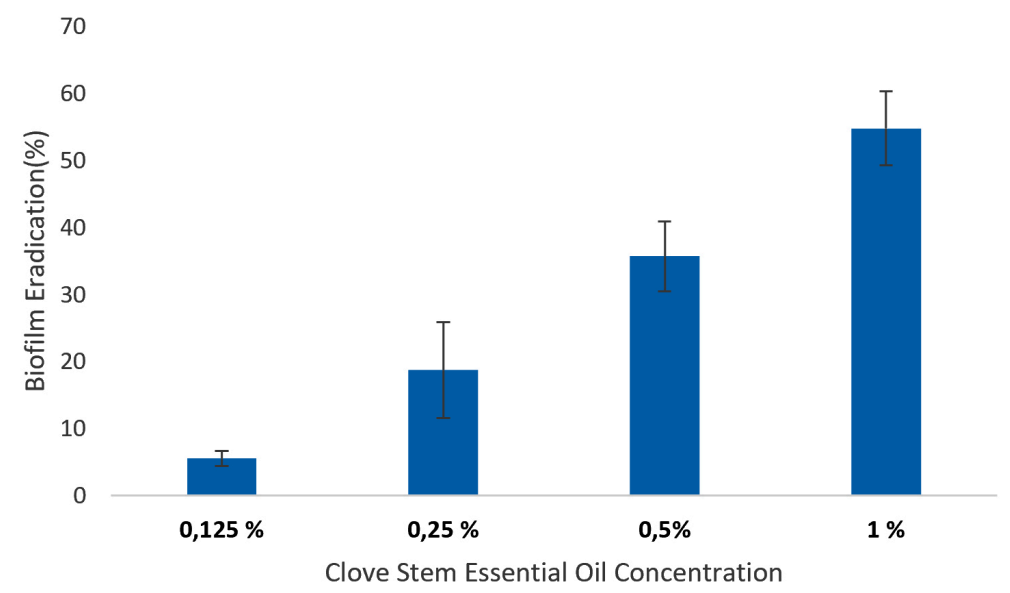

Fig. 3. MBEC assay of clove bud essential oil microemulsion. The highest concentration evaluated did not result in $100 \%$ biofilm elimination

Journal of Pure and Applied Microbiology 
lowest biofilm elimination occurred to the biofilm which had been pre-incubated 60 hours and had a high turbidity.

Fig. 5 and 6 show the MBIC assay results of clove bud and clove stem essential oil microemulsion. Clove bud essential oil microemulsion inhibited $67.81 \%$ of biofilm formation at $0.33 \%$ concentration, while clove stem essential oil microemulsion inhibited $57.5 \%$ of biofilm formation at $0.5 \%$ concentration. It resulted $\mathrm{MBIC}_{50 \%}$ value of clove bud and clove stem essential microemulsion were $0.33 \%$ and $0.5 \%$, respectively.
Fig. 7 and 8 show the result of clove bud and stem essential oil effect against extracellular protease activity and swarming motility of $P$. aeruginosa. At the highest concentration, both clove bud and stem essential oil microemulsion inhibited $\pm 45 \%$ of swarming motility but only inhibited $\pm 31 \%$ of extracellular protease activity.

\section{DISCUSSION}

Clove essential oil can be extracted from different parts of clove plant which resulted essential oil with different composition ${ }^{24}$. This research employed clove essential oil extracted

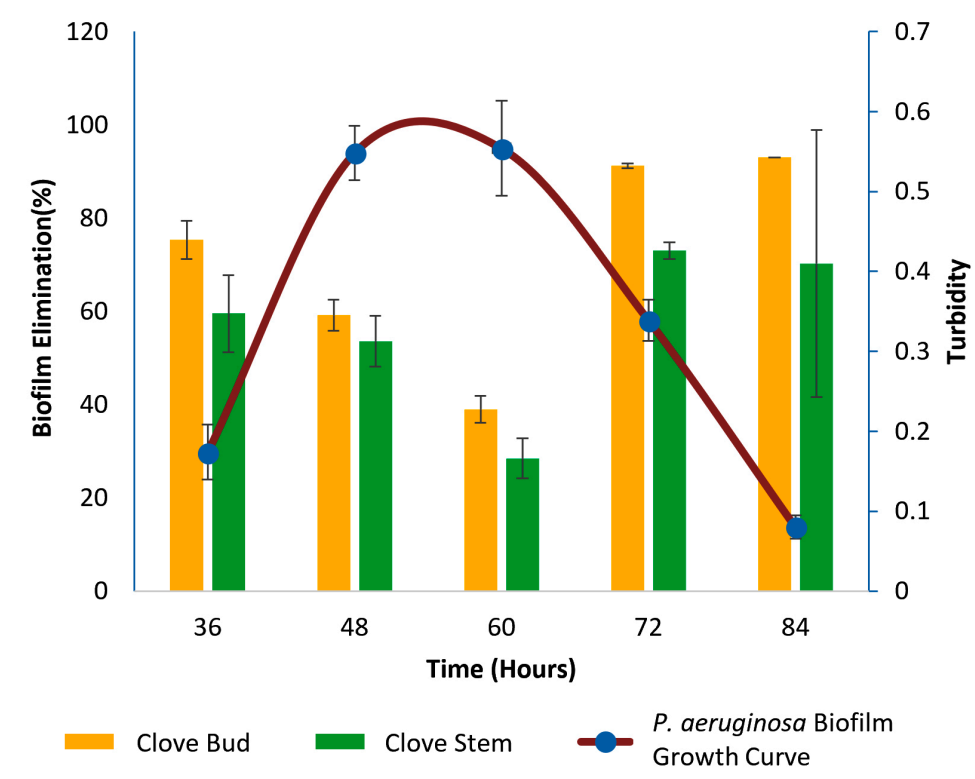

Fig. 4. P. aeruginosa biofilm elimination plotted to biofilm growth curve

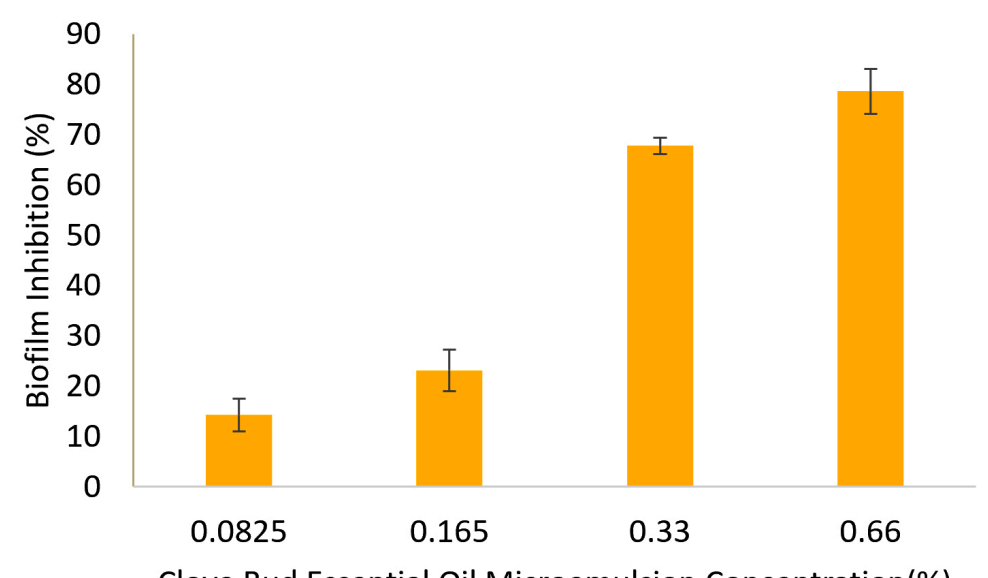

Clove Bud Essential Oil Microemulsion Concentration(\%)

Fig. 5. MBIC assay result of clove bud essential oil microemulsion. MBIC $50 \%$ was achieved at $1 \%$ of clove stem essential oil 
from clove bud and stem that produced relatively high yield of essential oil; $17-19 \%$ from bud and $6 \%$ from stem ${ }^{16}$. Both essential oil from different clove part were investigated for their ability to mitigate $P$. aeruginosa's biofilm formation.

Clove stem essential oil is known to contain higher concentration of eugenol (70-90\%) than clove bud essential oil $(70-80 \%)^{24}$. As the main antimicrobial component in clove, eugenol's concentration is expected to be directly related to the clove essential oil's antimicrobial activity. However, MIC assay showed that, compared with clove stem essential oil, clove bud essential oil has a lower MIC which indicated a higher antimicrobial capacity. This might be attributed to the antimicrobial activity of eugenyl acetate, which is an acetate compound derived from eugenol ${ }^{25}$. Clove bud essential oil contains up to $20 \%$ of eugenyl acetate, in contrast to clove stem essential oil which only contains less than $5 \%^{24}$. Supposedly, higher concentration of eugenyl acetate in clove bud essential oil is attributable to the increase of its antimicrobial activity.

MBEC assay showed that the highest concentration of both clove bud and clove stem essential oil did not result in $100 \%$ elimination of biofilm. This might be due to the protective structure of polymer matrix formed in biofilm. This matrix prevents antimicrobial compound to penetrate biofilm, which eventually kills the biofilm cells ${ }^{26}$.

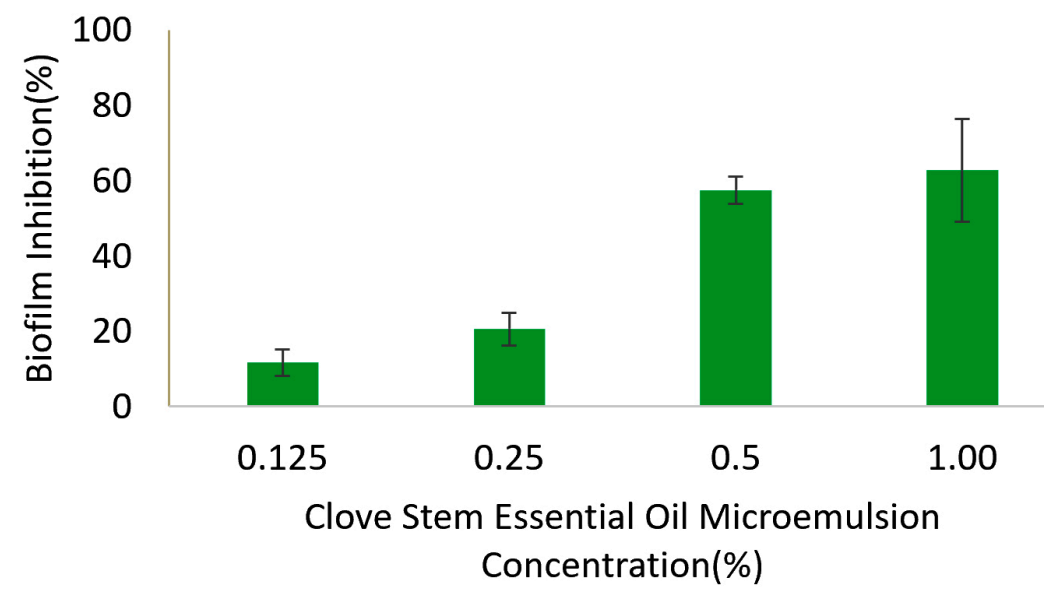

Fig. 6. MBIC value of clove stem essential oil microemulsion. MBIC50\% was achieved at $1 \%$ of clove stem essential oil

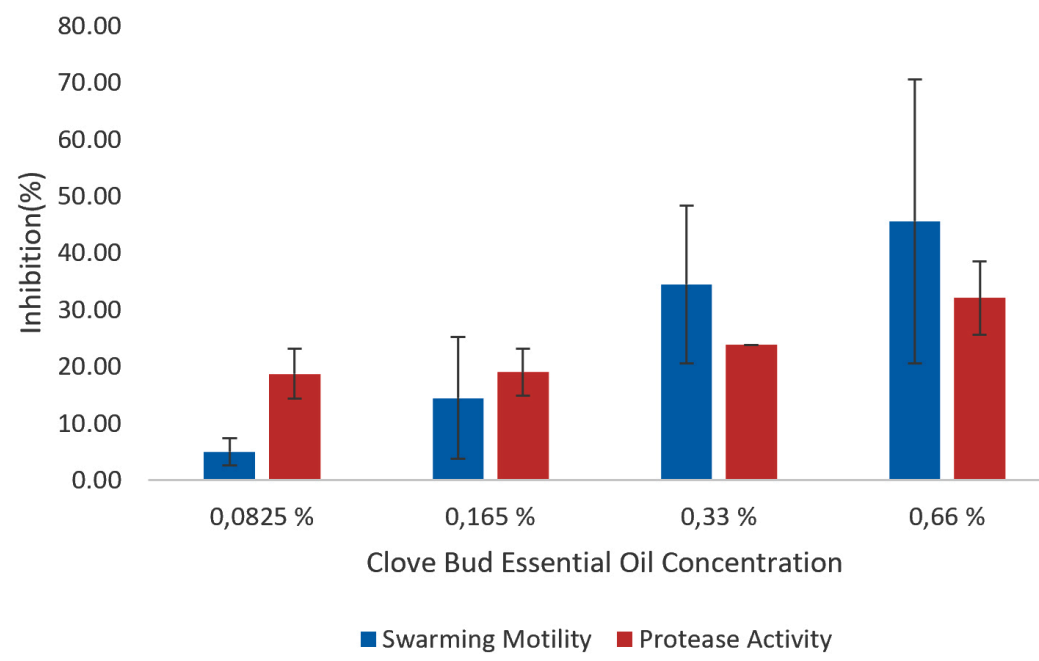

Fig. 7. P. aeruginosa quorum sensing inhibition by clove bud essential oil microemulsion 


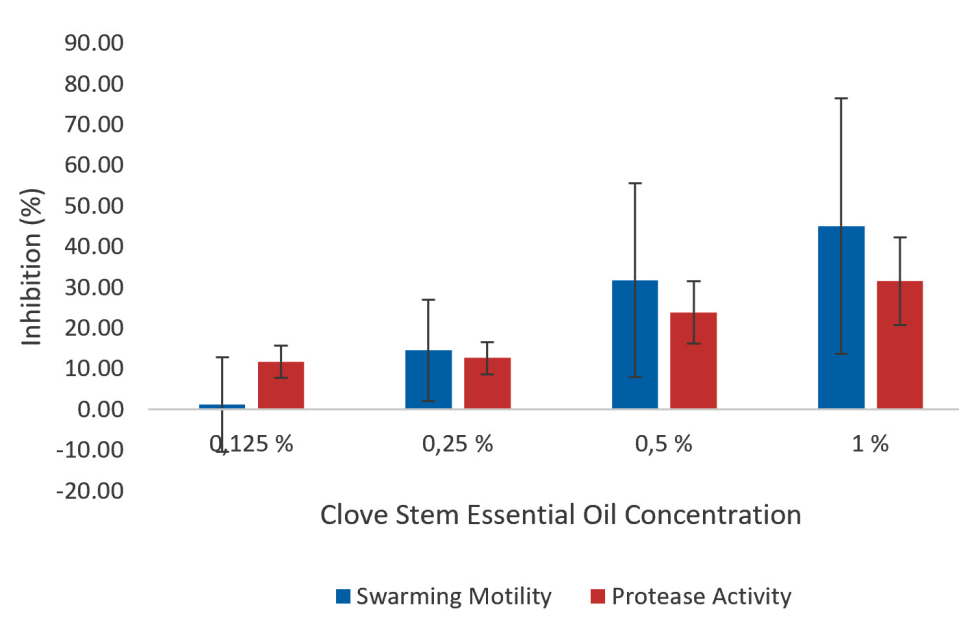

Fig. 8. P. aeruginosa quorum sensing inhibition by clove stem essential oil microemulsion

Clove essential oil was tested to biofilm with varying pre-incubation period to observe the correlation between biofilm growth and biofilm eradication. The result showed that the highest eradication rate occurred when biofilm growth was low, while the lowest eradication rate took place when biofilm growth was high. It indicated that there was a consistent correlation between biofilm eradication and biofilm growth which might be attributed to $P$. aeruginosa's biofilm formation cycle ${ }^{26}$.

At 84 hours of incubation, when the highest elimination of biofilm took place, the dispersion seemed to be occurred. Cell dispersion occurred because available nutrition has been used up and secondary metabolite which is hazardous for the cell has accumulated in biofilm ${ }^{26}$. As biofilm became thinner as the consequence of dispersion, it became more susceptible to antimicrobial compound. At 60 hours of incubation when the lowest biofilm elimination occurred, it subjected to optimal growth and maturation phase. Biofilm in this stage is enveloped in thick matrix and might have employed quorum sensing to activate additional defence mechanism to protect the cells from antimicrobial compound ${ }^{26}$. MBIC assay results indicated that biofilm inhibition was reliant on the clove essential oil concentration. The higher the concentration used; the more biofilm inhibition occurred. Both clove bud and stem essential oil did not cause $100 \%$ inhibition at the highest concentration analyzed in this study. It might be due to the initial cell number in the culture was high enough to completely colonize well's surface as the first step of biofilm formation ${ }^{22}$. The concentration of clove essential oil was not sufficient to inhibit all cells present in the culture.

Extracellular protease activity and swarming motility assay were conducted to observe the effect of clove essential oil to $P$. aeruginosa's QS process. Besides biofilm formation, these activities are among $P$. aeruginosa's phenotypes that are controlled by QS. Interference in extracellular protease activity and swarming motility suggest the anti-QS ability of clove essential oil that might correlate with its biofilm inhibitory activity. From the result, it can be observed that extracellular protease activity and swarming motility of $P$. aeruginosa were inhibited by clove essential oil less than $50 \%$. These values were even lower than biofilm inhibition assay results. This could be because eugenol, the main antimicrobial constituent in clove essential oil, does not effectively affecting the QS process. This result confirmed the studies reported by ${ }^{11,22}$ which indicated that pure eugenol was indeed not positively inhibited the quorum sensing on $P$. aeruginosa.

QS inhibition observed in this study might be conferred by other antimicrobial compounds which were present in minor concentration in clove essential oil24. Hence, inhibition activity observed in anti-QS assay as indicated by extracellular 
protease activity and swarming motility was lower than biofilm inhibition results.

The result also showed that swarming motility was more affected by clove essential oil than extracellular protease activity. This could be due to the variation in genetic regulation controlling these activities. QS system for extracellular protease activity was regulated by Las system encoded by LasB gene, while swarming motility was regulated by $\mathrm{Rhl}$ system, encoded by rhIAB gene $^{27}$. Differences in virulence factor affected by genetic regulation will influence its susceptibility to the overall antimicrobial compound's activity. Although the mechanism of QS inhibition is not yet elucidated, it can be concluded that clove essential oil extracted from bud and stem could interfere $P$. aeruginosa's QS process, particularly the ones regulated by $\mathrm{Rhl}$ system.

\section{CONCLUSION}

MIC value of clove bud and stem essential oils microemulsions were $0.33 \%$ and $0.5 \%$; MBIC $50 \%$ values for clove bud and stem essential oil were $0.33 \%$ and $0.5 \%$, however MBEC value for both clove essential oil could not be determined. This study suggested that clove bud and stem essential oil were more effective to inhibit biofilm formation rather than to eradicate it. In addition, $0.66 \%$ of clove bud essential oil and $1 \%$ of clove stem essential oil could inhibit extracellular protease activity and swarming motility of $P$. aeruginosa by $32.06 \%$ and $45.56 \%$ for clove bud, and $31.43 \%$ and $45 \%$ for clove stem, respectively.

\section{ACKNOWLEDGEMENTS}

The authors fully acknowledged Bandung Institute of Technology for the fund which made this study feasible to be conducted.

\section{CONFLICT OF INTEREST}

The authors declares that there is no conflict of interest.

\section{FUNDING}

This study was also supported by a grant from Bandung Institute of Technology on P3MI 2017 scheme.

\section{AUTHORS' CONTRIBUTION}

All authors listed have made a substantial contribution to the work and manuscript preparation. All authors approved this manuscript for publication.

\section{DATA AVAILABILITY}

All datasets obtained or studied during this study are incorporated in the manuscript.

\section{ETHICS STATEMENT}

This article does not contain any studies with human participants or animals performed by any of the authors.

\section{REFERENCES}

1. Popoola LT, Grema AJ, Latinwo GK, Gutti B, Balogun AS. Review: Corrosion problems during oil and gas production and its mitigation. Int J Ind Chem, 2013; 4(35): 1-15. https://doi.org/10.1186/2228-5547-4-35

2. Turkiewicz A, Brzeszcz J, Kapusta P. The application of biocides in the oil and gas industry. Nafta Gaz, 2013; 2: 103-111.

3. Abolahi A, Hamzah E, Ibrahim Z, Hashim S. Microbially influenced corrosion of steels by Pseudomonas aeruginosa. Corros Rev, 2014; 32(3-4): 129-141.

4. Ghazy EA, Elmokadem MT, Gadallah M, Mahmoud MN, Elsoud MMA. Combating biocorrosion induced by biofilm forming bacterial isolate Pseudomonas marginalis. Middle-East J Sci Res, 2012; 12(7): 10171025.

5. Davidson PM, Critzer FJ, Taylor TM. Naturally occurringantimicrobials for minimally processed foods. Annu Rev Food Sci T, 2013; 4: 163-190. https://doi. org/10.1146/annurev-food-030212-182535

6. Moon E, Kim Y, Cha D. Synergistic effect between clove oil and its major compounds and antibiotics against oral bacteria. Arch Oral Biol, 2011; 56(9): 907-916. https://doi.org/10.1016/j.archoralbio.2011.02.005

7. Uju D, Obioma P. Anticariogenic potentials of clove, tobacco and bitter kola. Asian Pac J Trop Med, 2011; 4(10): 814-818. https://doi.org/10.1016/S19957645(11)60200-9

8. Fei U, Yi-cheng D, Xing-qian E, Yu-ting D. Antibacterial effect of cinnamon oil combined with thyme or clove oil. Agric Sci China, 2011; 10(9): 1482-1487. https:// doi.org/10.1016/S1671-2927(11)60142-9

9. Guan W, Shufen L, Ruixiang Y,Tang S, Quan, C. Comparison of essential oils of clove buds extracted with supercritical carbon dioxide and three other traditional extraction methods. Food Chem, 2007; 101: 1558-1564. https://doi.org/10.1016/j. foodchem.2006.04.009

10. Edris A. Identification and absolute quantification of the major water-soluble aroma components isolated from the hydrosols of some aromatic plants. J Essn. Oil Bearing Plants, 2009; 12(2): 155-161. https://doi. org/10.1080/0972060X.2009.10643705 
11. Khan MSA, Zahin M, Hasan S, Husain FM, Ahmad I. Inhibition of quorum sensing regulated bacterial functions by plant essential oils with special reference to clove oil. Lett Appl Microbiol, 2009; 49(3): 354-360. https://doi.org/10.1111/j.1472-765X.2009.02666.x

12. Husain FM, Ahmad I, Asif M, Tahseen Q. Influence of clove oil on certain quorum-sensing regulated functions and biofilm of Pseudomonas aeruginosa and Aeromonas hydrophila. J Biosci, 2013; 38: 835-844. https://doi.org/10.1007/s12038-013-9385-9

13. Purwasena IA, Aditiawati P, Afinanisa Q, Siwi IK, Septiani H. Size Optimization of Lemongrass (Cymbopogon citratus) Essential Oil Nanoparticles as Antimicrobial Substance against Bacteria Isolated from South Sumatran Formation Water. Mater Res Express, 2019; 6(8): 0850h9. https://doi.org/10.1088/20531591/aac927

14. Okbi SYA, Mohamed DA, Hamed TE, Edris AE. Protective effect of clove oil and eugenol microemulsions on fatty liver and dyslipidemia as components of metabolic syndrome. J Med Food, 2014; 40(20): 1-8.

15. He S, Ren X, Lu Y, Zhang Y, Wang Y, Sun L. Microemulsification of clove essential oil improves its in vitro and in vivo control of Penicillium digitatum. Food Control, 2016; 65: 106-111. https://doi.org/10.1016/j. foodcont.2016.01.020

16. Babu AJ, Sundari AR, Indumathi J, Srujan RVN, Sravanthi M. Study on the antimicrobial activity and minimum inhibitory concentration of essential oils of spices. Vet World, 2011; 4(7): 311-316. https://doi.org/10.5455/ vetworld.4.311

17. Gupta S, Moulik SP, Lala S, Basu MK, Sanyal SK, Datta S. Designing and testing of an effective oil-in-water microemulsion drug delivery system for in vivo application. Drug Deliv, 2005; 12: 267-273. https:// doi.org/10.1080/10717540500176373

18. Anwer MK, Jamil S, Ibnouf EO, Shakeel F. Enhanced antibacterial effects of clove essential oil by nanoemulsion. J Oleo Sci, 2014; 63(4): 347-354. https://doi.org/10.5650/jos.ess13213

19. Adukwu EC, Allen SCH, Phillips CA. The anti-biofilm activity of lemongrass (Cymbopogon flexuosus) and grapefruit (Citrus paradisi) essential oils against five strains of Staphylococcus aureus. J Appl Microbiol, 2012; 113(5): 1217-1227. https://doi.org/10.1111/ j.1365-2672.2012.05418.x

20. Soumya EA, Saad IK, Hassan L, Ghizlane Z, Hind M, Adnane R. Carvacrol and thymol components inhibiting Pseudomonas aeruginosa adherence and biofilm formation. Afr J Microbiol Res, 2011; 5(20): 3229-3232. https://doi.org/10.5897/AJMR11.275

21. O’Tool G A. Microtiter dish biofilm formation assay. J Vis Exp, 2011; 47: 1-2. https://doi.org/10.3791/2437

22. Kalia M, Yadav VK, Singh PK, Sharma D, Pandey $H$, Narvi SS, Agarwal V. Effect of cinnamon oil on quorum sensing-controlled virulence factors and biofilm formation in Pseudomonas aeruginosa. PLOS ONE, 2015; 10(8): 1-18. https://doi.org/10.1371/journal. pone.0135495

23 O'May C, Tufenkji N. The swarming motility of Pseudomonas aeruginosa is blocked by cranberry proanthocyanidins and other tannin-containing materials. Appl Env Microbiol, 2011; 77(9): 3062. https://doi.org/10.1128/AEM.02677-10

24. Razafimamonjison $\mathrm{G}$, Jahiel $\mathrm{M}$, Duclos $\mathrm{T}$, Ramanoelina P, Fawbush F, Thu P. Bud, leaf and stem essential oil composition of clove (Syzygium aromaticum L.) from Indonesia, Madagascar and Zanzibar. Nat Prod Commun, 2012; 8: 1-7.

25. Vanin $A B$, Orlando T, Piazza SP, Puton BMS, Cansian $\mathrm{RL}$, Oliveira D, Paroul N. Antimicrobial and antioxidant activities of clove essential oil and eugenyl acetate produced by enzymatic esterification. Appl Biochem Biotechnol, 2014; 174(4): 1286-1298. https://doi. org/10.1007/s12010-014-1113-x

26. Rasamiravaka T, Labtani Q, Duez P, Jaziri ME. Review Article: The formation of biofilms by Pseudomonas aeruginosa: a review of the natural and synthetic compounds interfering with control mechanisms. BioMed Res Int, 2015; 1-5. https://doi. org/10.1155/2015/759348

27. Lee J, Zhang L. Review: The Hierarchy quorum sensing network in Pseudomonas aeruginosa. Protein Cell, 2015; 6(1):26-41. https://doi.org/10.1007/s13238014-0100-x 